\title{
BDM Dark Matter: CDM with a core profile and a free streaming scale
}

\author{
A. de la Macorra \\ Instituto de Física, Universidad Nacional Autonoma de Mexico, \\ Apdo. Postal 20-364, 01000 México D.F., México \\ Part of the Collaboration Instituto Avanzado de Cosmologia
}

\begin{abstract}
We present a new dark matter model BDM which is an hybrid between hot dark matter HDM and cold dark matter CDM, in which the BDM particles behave as HDM above the energy scale $E_{c}$ and as CDM below this scale. Evolution of structure formation is similar to that of CDM model but BDM predicts a nonvanishing free streaming $\lambda_{f s}$ scale and a inner galaxy core radius $r_{\text {core }}$, both quantities determined in terms of a single parameter $E_{c}$, which corresponds to the phase transition energy scale of the subjacent elementary particle model. For energies above $E_{c}$ or for a scale factor $a$ smaller then $a_{c}$, with $a<a_{c}<a_{e q}$, the particles are massless and $\rho$ redshifts as radiation. However, once the energy becomes $E \leq E_{c}$ or $a>a_{c}$ then the BDM particles acquire a large mass through a non perturbative mechanism, as baryons do, and $\rho$ redshifts as matter with the particles having a vanishing velocity. Typical energies are $E_{c}=O(10-100) \mathrm{eV}$ giving a $\lambda_{f_{s}} \propto E_{c}^{-4 / 3} \lesssim \mathrm{Mpc}$ and $M_{f s} \propto E_{c}^{-4} \lesssim 10^{9} M \odot$. A $\lambda_{f s} \neq 0, r_{\text {core }} \neq 0$ help to resolve some of the shortcomings of CDM such as overabundance substructure in CDM halos and numerical fit to rotation curves in dwarf spheroidal and LSB galaxies. Finally, our BDM model and the phase transition scale $E_{c}$ can be derived from particle physics.
\end{abstract}

\section{INTRODUCTION}

The understanding of our universe has received a great deal of attention in recent times. Cosmological data such as large scale structure [1], SN1a [2] and CMB [3] are consistent with the concordance $\Lambda C D M$ model, with $\Omega_{d m o}=0.22, \Omega_{\Lambda o}=74, \Omega_{b o}=0.04$ with $h_{o}=0.71$. Even though the simple concordance model describes well our universe the nature of dark energy DE and dark matter DM, which account for up to $96 \%$ of the energy content, is not well understood. A large number of candidates have been proposed for DM of which cold dark matter (CDM) has been the most popular. CDM model has been successful on large scales in explaining structure formation in the early universe as well as abundances of galaxy clusters [1]. However, CDM predicts steeply cusped density profiles and causing a large fraction of haloes to survive as substructure inside larger haloes $[4,5]$. These characteristics of CDM haloes, however, seem to disagree with a number of observations. The number of sub-haloes around a typical Milky Way galaxy, as identified by satellite galaxies, is an order of magnitude smaller than predicted by CDM [6] and the observed rotation curves for dwarf spheriodal dSph and low surface brightness (LSB) galaxies seem to indicate that their dark matter haloes have constant density cores $[7,8]$ instead of steep cusps as predicted by the NFW profile. Low surface brightness galaxies are diffuse, low luminosity systems, with a total mass believed to be dominated by their host dark matter halos [9]. Assuming that LSB galaxies are in dynamical equilibrium, the stars act as tracers of the gravitational potential, and can therefore be used as a probe of the dark matter density profile [10]. Much better fits to dSph and LSB observations are found when using a cored halo model [11]. Cored halos have a mass-density that remains at an approximately constant value towards the center.
It has been argued that the sub-structure and core problems might be solved in a NFW profile once additional baryonic physics are taken into account such as reionization and supernova feedback. This feedback may help to suppress star formation and to decrease central densities in low-mass dark matter haloes [12]. However, even tough these processes may help to solve the problem with the over-abundance of satellite galaxies, the suggestion that feedback processes can actually destroy steep central cusps seems somewhat contrived in detailed simulations [13]. Due to these discrepancies in CDM, numerous alternatives to the CDM paradigm have been proposed. These include broken scale-invariance [14], warm dark matter [15], scalar field dark matter [16], and various sorts of self-interacting or annihilating dark matter [17]. However, these alternatives are unable to solve both problems simultaneously.

Here we propose a new version of dark matter, well motivated from particle physics, which predicts simultaneously a cut in the substructure formation and cored center galaxies. The model simply consist of particles that at high energy densities are massless relativistic particles with a velocity of light, $v=c$, but at low densities they acquire a large mass, due to nonperturbative quantum field effects, and become non relativistic with a vanishing (small) dispersion velocity. We will name this type of dark matter BDM, from bound states dark matter. The name is motivated by the particle physics model, discussed in section III, but we would like to stress out that the cosmological properties of BDM do not depend on this particle model but on the different behavior of the BDM particles. The phase transition energy density is defined $\rho_{c} \equiv E_{c}^{4}$ and its value can be determined theoretical by the particle physics model or phenomenological by consistency with the cosmological data.

There are two natural places where one encounters high energy densities for dark matter. Firstly, at early 
times when the universe is hot and dense, and secondly in the center of galaxies. From a cosmological point of view, our BDM has then two clearly distinct behaviors: one as hot dark matter HDM at high energies $\rho_{c}<\rho_{b d m} \sim$ $a(t)^{-4}$ with $\Omega_{b d m}$ being constant and another as CDM for energies smaller than $\rho_{c}>\rho_{b d m} \sim a(t)^{-3}$. The transition takes place at $a_{c}$ and for $a<a_{c}$ the BDM particles are relativistic but for $a>a_{c}$ they become non-relativistic. If $\mathrm{BDM}$ accounts for all $\mathrm{DM}$ than the redshift $a_{c}$, at which we have the phase transition $\rho\left(a_{c}\right) \equiv \rho_{c}$, must be smaller than the matter-radiation equality $a_{e q}$ and $\rho_{c} \geq \rho_{e q}$. From constraints on extra relativistic energy density at the time of nucleosynthesis we will have an upper limit for $a_{c}$ or equivalently a lower limit for $\rho_{c}$.

Since the particles of BDM travel at the speed of light at energy densities above $\rho_{c}$ there will be a cut at small scales in the power spectrum and BDM will erase inhomogeneities and inhibit structure formation for scales below the free streaming scale $\lambda_{f s}$. This property is similar to that of WDM and it predicts lower number of substructure in DM halos as a CDM model, welcome by the data. The value of $\lambda_{f s}$ will depend only on the phase transition energy scale $E_{c} \equiv \rho_{c}^{1 / 4}$. Once the universe expands and $\rho_{b d m}$ drops to values smaller than $\rho_{c}$, the speed of the BDM particles vanishes and these particles will then be CDM until present time.

Once DM dominates the universe, structure formation is effective, our BDM particles are cold and we expect a standard CDM inhomogeneities growth but with the a cutoff $\lambda_{f s}$ in the power spectrum. The average energy densities in halos is of the order $10^{5} \rho_{o}$ and as long as $\rho_{g}<\rho_{c}$ we expect a standard CDM galaxy profile, which may be given by the NFW profile. However, once we approach the center of the galaxy the energy density increases and once it reaches the point $\rho_{g}=\rho_{c}$ we encounter a phase transition for the BDM particles and they become massless again. We identify this energy density of the galaxy with the core energy density $\rho_{\text {core }}=\rho_{c}$ at a core galaxy radius $r=r_{\text {core }}$. Inside $r<r_{\text {core }}$ the $\mathrm{BDM}$ particles are relativistic and the DM energy density inside the radius $r_{\text {core }}$ remains constant avoiding a galactic cusp. Of course we would expect a smooth transition region between these two distinct behaviors but the effect of considering the thickness of this transition region is a small and we will not consider it here. As for the free streaming scale $\lambda_{f s}$, the size of $r_{\text {core }}$ depends only on $E_{c}$.

Therefore, our BDM model predicts a free streaming scale $\lambda_{f s}$, a cut off in the power spectrum and a galaxy core radius $r_{\text {core }}$ all derived in terms of a single parameter $E_{c}$. The value of $E_{c}$ is determined by the particle physics model and constraint by the dark matter properties derived from cosmology. Furthermore, since $E_{c}$ gives the phase transition scale of a particle physics model further phenomenological consequences could arise. We present in section II the cosmological relevant properties of BDM model and in section III the particle physics model giving BDM. Finally, we present in section IV our conclusions.

\section{BDM COSMOLOGY}

We consider a standard FRW model universe with dark energy and dark matter. The dark matter proposed here is motivated in section III from particle physics and it has a phase transition at an energy scale $E_{c}$ or equivalently at an energy density $\rho_{c} \equiv E_{c}^{4}$. For energy densities larger than $\rho_{c}$ the particles have a peculiar velocity $v=c=1$ and behaves as relativistic dark matter while for $\rho_{b d m}<$ $\rho_{c}$ the velocity vanishes and $\rho_{b d m}$ behaves as cold dark matter. We encounter naturally two regions in cosmology with a high $\rho_{b d m}$. The first one is at early times when the universe is hot and as long as $\rho_{b d m}(a)>\rho_{c}\left(a_{c}\right)$, where $a_{c}$ is the transition redshfit, and the second region is in the center of galaxies for a radius $r<r_{\text {core }}$ with $\rho_{b d m}(r)>$ $\rho\left(r_{\text {core }}\right)=\rho_{c}$.

The cosmological evolution of the BDM energy density and the peculiar velocity in terms of the scale factor $a(t)$ is then given by

$$
\begin{array}{r}
\rho_{b d m}(a)=\rho_{c}\left(\frac{a_{c}}{a}\right)^{4} \geq \rho_{c}, \quad v=1, \quad a \leq a_{c} \\
\rho_{b d m}(a)=\rho_{b d m o}\left(\frac{a_{o}}{a}\right)^{3} \leq \rho_{c}, \quad v=0, \quad a \geq a_{c}
\end{array}
$$

where the subscript $o$ is at present time and

$$
\rho_{c} \equiv E_{c}^{4} \equiv \rho_{b d m}\left(a_{c}\right)
$$

is the critical or phase transition energy density. In

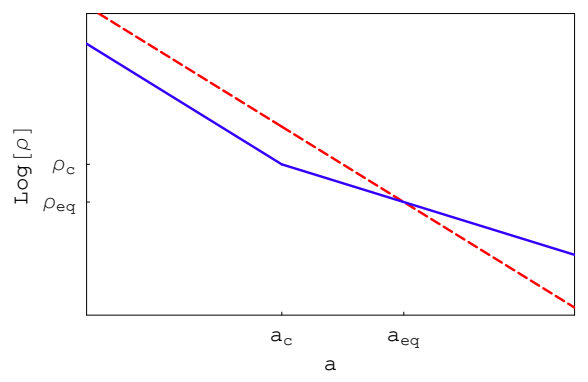

FIG. 1: We show the behaviour of BDM and radiation (solid (blue) and dotted (red), respectively) and the transition scale $a_{c}<a_{e q}$ where BDM changes its evolution from $\rho \propto 1 / a^{3}$ to $\rho \propto 1 / a^{4}$.

terms of a convenient phase transition scale $E_{c}=O(10-$ 100) $\mathrm{eV}$, derived from the particle physics model [18] (c.f. sec.III), we have

$$
\rho_{c}=\left(\frac{E_{c}}{60 \mathrm{eV}}\right)^{4} 10^{17} \rho_{o}=4 \times 10^{10}\left(\frac{E_{c}}{60 \mathrm{eV}}\right)^{4} M_{\odot} / p c^{3} .
$$

If we require $\rho_{b d m}$ to account for all dark matter the scale factor $a_{c}$ must be smaller than $a_{e q}$, the matter-radiation equivalence scale factor. Of course it also possible that $\rho_{b d m}$ accounts only partially for DM in which case $a_{c}$ could be larger than $a_{e q}$. We can expressed $a_{c}$ in terms 


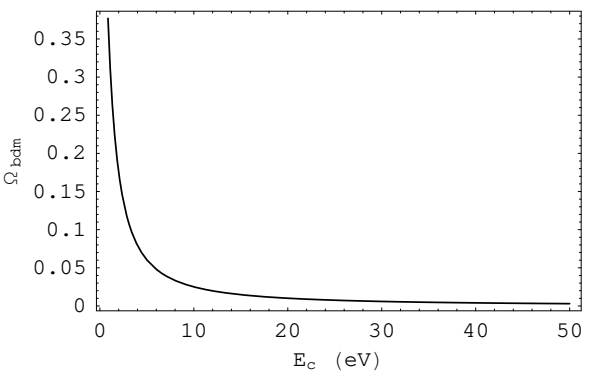

FIG. 2: We show the dependence of $\Omega_{b d m}$ as a function of $E_{c}$. $\Omega_{b d m}$ is smaller for larger $E_{c}$ and it is constant for $a<a_{c}$.

of the transition energy density as

$$
a_{c}=\left(\frac{\rho_{b d m o}}{\rho_{c}}\right)^{1 / 3}=\left(\frac{3 H_{o}^{2} \Omega_{b d m o}}{E_{c}^{4}}\right)^{1 / 3}
$$

where we have taken $a_{o}=1, \Omega_{r o}$ is the relativistic energy density and $\Omega_{m o}=\Omega_{b d m o}+\Omega_{b o}$ the total matter at present time and $a_{e q}=\Omega_{r o} / \Omega_{m o}$. For $a_{c} \leq a_{e q}$, eq.(5) gives $E_{c}>0.9 \mathrm{eV}$ with $E_{e q}=0.5 \mathrm{eV}$ and $\Omega_{b d m}(a)$ remains constant for $a<a_{c}$ since the universe is dominated by radiation and $\rho_{b d m}$ behaves in this region also as a relativistic fluid. Therefore, $\Omega_{b d m}\left(a \leq a_{c}\right)=\Omega_{b d m}\left(a_{c}\right) \equiv$ $\Omega_{b d m c}$ is constant and in terms of the transition scale we have

$$
\begin{aligned}
\Omega_{b d m c} & =\frac{\rho_{c}}{3 H_{c}^{2}}=\frac{\Omega_{b d m o}}{\Omega_{m o}}\left(\frac{q}{1+q}\right)=0.005\left(\frac{60 e V}{E_{c}}\right)^{4 / 3} \\
q\left(E_{c}\right) & \equiv \frac{a_{c}}{a_{e q}}=\frac{\Omega_{m o}}{\Omega_{r o}}\left(\frac{\rho_{b d m o}}{\rho_{c}}\right)^{1 / 3}=0.004\left(\frac{60 e V}{E_{c}}\right)^{4 / 3}(6)
\end{aligned}
$$

with $3 H_{c}^{2}=\rho_{T c}=\rho_{r}\left(a_{c}\right)+\rho_{b d m}\left(a_{c}\right)+\rho_{b}\left(a_{c}\right)$, $\Omega_{b d m o} / \Omega_{m o}=0.22 / 0.26$ and $q \sim 10^{-3} \ll 1$. The amount of extra energy density is constraint by nucleosynthesis "NS". The upper bound is $\Omega_{\text {extra }}(\mathrm{MeV})<0.1-0.2$ [20] and from eq.(6) we have then the constraint $E_{c}>$ $(3.3-1.8) e V$ and $q=a_{c} / a_{e q}<(0.13-0.3)$, respectively. We see that NS constraint allows the transition redshift $a_{c}$ or $E_{c}$ to be quite close to the matter-radiation equivalence values but with $E_{c}>E_{e q}$ and $a_{c}<a_{e q}$. We show in fig(2) the evolution of $\rho(a)$ with the pase transition at $a_{c}$. In fig.(1) we show the dependence of $\Omega_{b d m}$ as a function of $E_{c}$ for $a<a_{c}$.

It is well know that a HDM model has a cut in the power spectrum and does not allowed structure to form below the free streaming scale $\lambda_{f s}$ containing a mass $M_{f s}=4 \pi \rho_{o}\left(\lambda_{f s} / 2\right)^{3} / 3$. A large $M_{f s}>10^{12} M_{\odot}$, as for neutrinos, is ruled out by structure formation but a smaller $M_{f s} \simeq 10^{9} M_{\odot}$ would help to solve the problem of having too much substructure in a CDM scenario. The free streaming scale $\lambda_{f s}$ is given by

$$
\begin{aligned}
\lambda_{f s}(t) & =a(t) \int_{0}^{t} d t^{\prime} \frac{v\left(t^{\prime}\right)}{a\left(t^{\prime}\right)} \\
& =a(t) \int_{0}^{a_{c}} \frac{d a}{a^{2} H}=\frac{a(t)}{H_{c} a_{c}}
\end{aligned}
$$

where we have used $d t=d a / a H$ and that the velocity of the BDM is given by $v=1$ for $a \leq a_{c}$ and $v=0$ for $a>a_{c}$. Using eq.(5) with $3 H_{c}^{2}\left(a_{c}\right)=\rho_{T c}\left(a_{c}\right)=\rho_{c} / \Omega_{b d m c}$ and eq.(7) we get a free streaming scale

$$
\begin{aligned}
\lambda_{f s}\left(E_{c}\right) & =\frac{\sqrt{\Omega_{r o}}}{H_{o} \Omega_{m o}} \frac{q}{\sqrt{1+q}}=\frac{\rho_{b d m o}^{1 / 3} E_{c}^{-4 / 3}}{H_{o} \sqrt{\Omega_{r o}} \sqrt{1+q}} \\
& \simeq\left(\frac{60 e V}{E_{c}}\right)^{4 / 3} 0.4 \mathrm{Mpc}
\end{aligned}
$$

and a contained mass within a radius $\lambda_{f s} / 2$

$$
M_{f s}\left(E_{c}\right) \equiv \frac{4 \pi \rho_{o}}{3}\left(\frac{\lambda_{f s}}{2}\right)^{3}=\left(\frac{60 e V}{E_{c}}\right)^{4} 5.1 \times 10^{9} M_{\odot}
$$

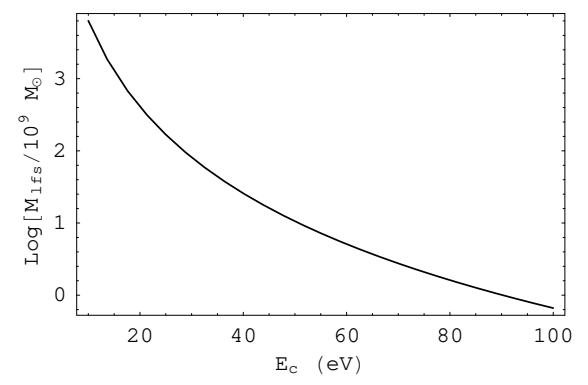

FIG. 3: We show the dependence of $M_{f s}$ as a function of $E_{c}$ and the larger $E_{c}$ the smaller $M_{f s}$.

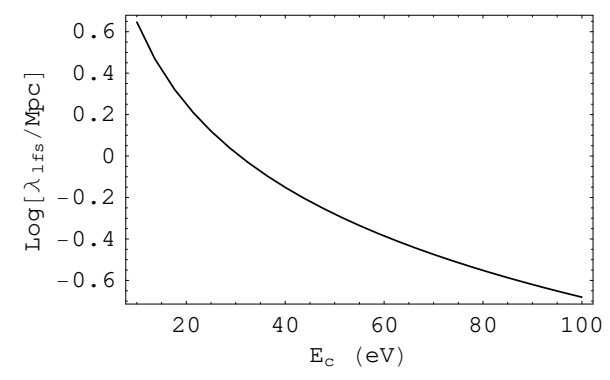

FIG. 4: We show the dependence of $\lambda_{f s}$ as a function of $E_{c}$ and the larger $E_{c}$ the smaller $\lambda_{f s}$.

We see that $\lambda_{f s}$ and $M_{f s}$ only depend on $E_{c}$ and are inversely proportional to it. The larger the scale $E_{c}$ (or $\rho_{c}$ ) the smaller $\lambda_{f s}, M_{f s}$ will be. The value $E_{c} \sim 60 \mathrm{eV}$ gives precisely a $M_{f s}$ of the order of $10^{9} M_{\odot}$ as required to inhibit extra substructure in halo but small enough to allow for small galaxies to be produced. For scales $\lambda<\lambda_{f s}$ there will be little power on the spectrum. The constraint to have $M_{f s}<10^{10} M_{\odot}$ gives a lower bound to $E_{c}$, with $E_{c}>50 \mathrm{eV}$ or $\rho_{c}>(50 \mathrm{eV})^{4}$. In fig.(3) and (4) we show the dependence of $M_{f s}, \lambda_{f s}$ as a function of $E_{c}$.

In a spherical DM distribution the rotation velocity vel can be determine straightforward by $\nabla^{2} \Phi=4 \pi \rho$ with 
$\Phi=-G M(r) / r$, giving

$$
\text { vel }=\sqrt{\frac{G M(r)}{r}}
$$

and $M=4 \pi \int \rho(r) r^{2} d r$. The Jeans scale radius $r_{J}\left(E_{c}\right)=\lambda_{J} / 2$ is given by

$$
r_{J}\left(E_{c}\right)=\pi \sqrt{\frac{v_{s}^{2}}{4 \pi G \rho}}=\sqrt{\frac{2}{3}} \frac{\pi}{E_{c}^{2}}=\left(\frac{60 e V}{E_{c}}\right)^{2} 11 \mathrm{pc}
$$

where we have used $\rho=\rho_{c}=E_{c}^{4}$, the sound speed for a relativistic fluid $v_{s}^{2}=1 / 3$ and $8 \pi G=1$. The Jeans scale implies that inhomogeneities at scales below $\lambda_{J}$ inside a galaxy are erased by the free streaming of the particles, i.e. we have a constant inner galaxy core. The Jeans radius is the maximum size for an inner core which co-

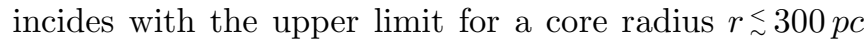
[9] giving a scale $E_{c} \gtrsim 10 \mathrm{eV}$. It is well known that in a cosmological scenario the Jeans scale $r_{J}$ is larger than the Hubble radius $d_{H}=1 / H$ for $a<a_{e q}$ and therefore structure formation does not form until a matter domination universe. However, in a galactic scenario $r_{J}$ gives the largest possible radius for a central core region. In-

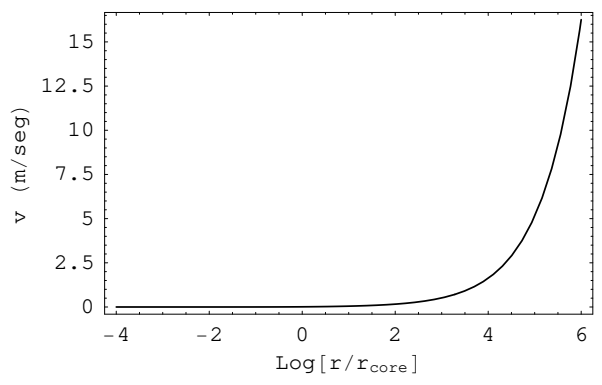

FIG. 5: We show the inner circular velocity of $v$ as a function of $r / r_{\text {core }}$ for a LSB type galaxy with $\rho_{s}=5 \times 10^{4} \rho_{o}, r_{s}=$ $3 h^{-1} k p c$.

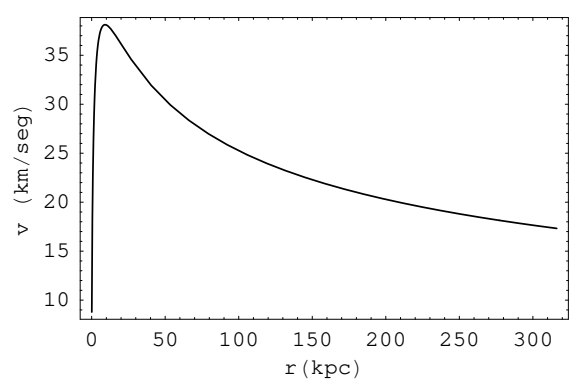

FIG. 6: We show the circular velocity of $v$ as a function of $r$ for a LSB type galaxy with $\rho_{s}=5 \times 10^{4} \rho_{o}, r_{s}=3 h^{-1} k p c$.

dependent of the galaxy density profile we can estimate the contained cored mass as a function of the core radius $r_{\text {core }}$ using eq.(4)

$$
M_{\text {core }}=\frac{4 \pi \rho_{c} r_{\text {core }}^{3}}{3}=40\left(\frac{E_{c}}{60 e V}\right)^{4}\left(\frac{r_{\text {core }}}{0.001 p c}\right)^{3} M_{\odot} .
$$

We see from eq.(13) that a contained mass $M_{\text {core }}=$ $O(1) M_{\odot}$ requires a small $r_{\text {core }}=O\left(10^{-3}\right) p c$ for a $E_{c} \simeq$ $O(10-100) \mathrm{eV}$. Since $r_{\text {core }}$ is small we expect a small deviation from a CDM profile. There are different galaxy profiles in the literature. The standard CDM profile is given by the NFW $\rho_{N F W}=\rho_{s} /\left[\left(r / r_{s}\right)\left(1+r / r_{s}\right)^{2}\right][4]$ while a core galaxy may be fit with $\rho(r)=\rho_{b} /[(1+$ $\left.\left.r / r_{b}\right)\left(1+\left(r / r_{b}\right)^{2}\right)\right][7]$ with $\rho_{b}, r_{b}$ the core density and radius, respectively. The NFW profile has a cuspy inner region with $\rho_{N F W}$ diverging in the center of the galaxy with an inner slope $\alpha=-1\left(\rho_{g} \propto r^{\alpha}\right)$ and $\rho_{s}, r_{s}$ are galaxy dependent parameters. For typical LSB galaxies one has $\rho_{s} \simeq 10^{4} \rho_{o}, r_{s} \simeq 3 h^{-1} k p c$ [4]. As mentioned in the introduction, a core inner region with constant $\rho$ and a slope $\alpha<-1 / 2$ seems to be preferred by $\mathrm{dSph}$ and LSB galaxies [9]. The core region is reached at $r \leq r_{\text {core }}$ when the galaxy energy density reaches the value $\rho_{c}$,

$$
\rho_{g}(r) \simeq \rho_{\text {core }}\left(r_{\text {core }}\right) \equiv \rho_{c} .
$$

The size of the core radius $r_{\text {core }}$ depends on the choice of the galaxy profile. Since our BDM behaves as CDM for $\rho<\rho_{c}$ as long as the density of the galaxy is $\rho_{g}<\rho_{c}$ we expect to have a NFW type profile. Therefore, a possible $\mathrm{BDM}$ profile is given by a cored CDM profile as

$$
\rho_{g}(r)=\frac{\rho_{s}}{\left(r_{\text {core }} / r_{s}+r / r_{s}\right)\left(1+r / r_{s}\right)^{2}}
$$

with $r_{\text {core }} \ll r_{s}$. This profile coincides with $\rho_{N F W}$ at large radius but has a core inner region at $r=r_{\text {core }}$ with $\rho_{g}\left(r_{\text {core }}\right)=\rho_{c}=\rho_{s} r_{s} / 2 r_{\text {core }}$ giving a core radius

$$
r_{\text {core }}\left(E_{c}\right) \equiv \frac{\rho_{s} r_{s}}{2 \rho_{c}}=\frac{\rho_{s} r_{s}}{2 E_{c}^{4}}
$$

The slope is

$$
\alpha \equiv \frac{d \log [\rho]}{d \log [r]}=-\frac{r / r_{\text {core }}\left(1+3 r / r_{s}+2 r_{\text {core }} / r_{s}\right)}{\left(1+r / r_{\text {core }}\right)\left(1+r / r_{s}\right)}
$$

and takes the values $\alpha=(0,-1 / 2,-1,-2,-3)$ for $r=$ $\left(0, r_{\text {core }}, r_{\text {core }} \ll r \ll r_{s}, r_{s}, r_{s} \ll r\right)$ respectively. For values of $r \ll r_{s}$, eqs.(11) and (15) gives in terms of $r^{\prime} \equiv r / r_{\text {core }}$ a central mass, circular velocity and a slope

$$
\begin{aligned}
M\left(r^{\prime}\right) & \simeq 8 \pi \rho_{c} r_{\text {core }}^{3}\left(\frac{r^{\prime 2}}{2}-r^{\prime}+\log \left[1+r^{\prime}\right]\right) \\
v\left(r^{\prime}\right) & \simeq r_{\text {core }} \sqrt{\rho_{c}}\left(\frac{r^{\prime}}{2}-1+\frac{\log \left[1+r^{\prime}\right]}{r^{\prime}}\right)^{1 / 2} \\
\alpha\left(r^{\prime}\right) & \simeq-\frac{r^{\prime}}{1+r^{\prime}} .
\end{aligned}
$$

Notice that from eqs.(8) and (16) we can express $\lambda_{f s}$ in terms of $r_{\text {core }}$

$$
\lambda_{f s}=b r_{\text {core }}^{1 / 3}
$$

with $b=\left(2 \sqrt{\rho_{b d m o}} / \rho_{s} r_{s}\right)^{1 / 3} /\left(H_{o} \sqrt{\Omega_{r_{o}}(1+q)}\right)$ a proportionality constant, showing explicitly the interconnection 
of $\lambda_{f s}$ and $r_{\text {core }}$. In fig.(5) we show the central circular velocity as a function of $r / r_{\text {core }}$ while in fig. (6) we plot the complete circular velocity as a function of $r(k p c)$. In fig.(7) we show the central slope profile as a function of $r / r_{\text {core }}$ while in fig. (8) we have the complete $\alpha$ as a function of $r(k p c)$.

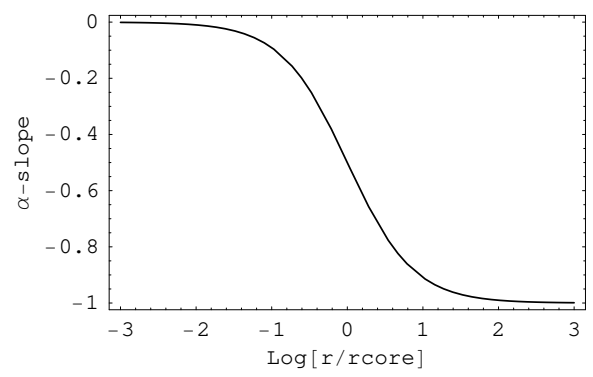

FIG. 7: We show the inner slope of the galaxy profile in eq.(15) as a function of $r / r_{\text {core }}$.

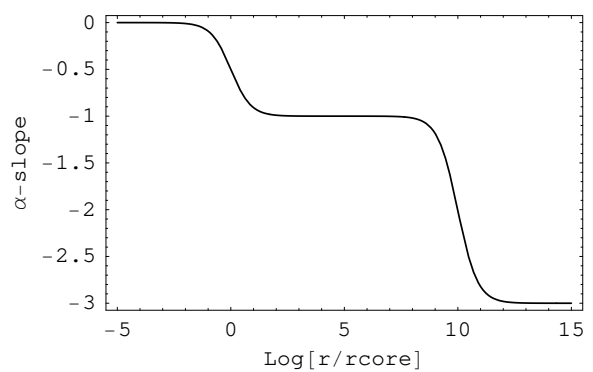

FIG. 8: We show the complete slope of the galaxy profile in eq.(15).

\section{PARTICLE MODEL}

In particle physics there are two different dynamically ways to generate a particle mass, namely the Higgs mechanism and the a non-perturbative gauge mechanism. In the SM the elementary particles (quarks, electrons, neutrinos) get their mass by the interaction with the Higgs field. The dynamically evolution of the Higgs field implies that at high energies all SM masses vanish but once the Higgs settles into the minimum of its potential, at the electroweak scale $E_{e w}=O(100 \mathrm{GeV})$, it acquires a non vanishing vacuum value giving a mass to the SM particles. Therefore, the mass of the fundamental particles vanishes at high energies and are non zero below the phase transition scale $E_{e w}$. On the other hand, the nonperturbative gauge mechanism is based on the strength of gauge interaction. The strength of the coupling constant "g" depends on the energy as

$$
g^{-2}(E)=g_{i}^{-2}+8 \pi^{2} b \log \left[\frac{E}{E_{i}}\right]
$$

with $g_{i}=g\left(E_{i}\right)$ and $b$ counts the number of elementary particles "Q" charged under the group. If $b>0$, as for the strong force QCD with $S U\left(N_{c}=3\right), N_{f}=6\left(N_{c}, N_{f}\right.$ are the number of colors and flavors, respectively) one has $b=\left(11 N_{c}-2 N_{f}\right) / 3=7$, the gauge coupling constant increases with decreasing energy $E$ and we have a nonabelian asymptotic free gauge group. The condensation or phase transition scale is defined as the energy when the coupling constant becomes strong, $g(E) \gg 1$, and from eq.(22) we have

$$
E_{c}=E_{i} e^{-8 \pi^{2} / b g_{i}^{2}} .
$$

The fact that $E_{c}$ is exponentially suppressed compared to $E_{i}$ allows as to understand why $E_{c}$ can be much smaller then the initial $E_{i}$ which may be the Planck, Inflation or Unification scale. The scale $E_{c}$ sets a phase transition scale where above $E_{c}$ the elementary particles "Q" (e.g. quarks in QCD) are (nearly) massless and below $E_{c}$ the strong force binds these elementary fields together forming neutral bounds states such as mesons and baryons in QCD. The order of magnitude of the mass of these particles is

$$
m_{B S}=d E_{c}
$$

with $d=O(1)$ a proportionality constant and a Compton wave length

$$
\lambda_{\text {com }}=\frac{1}{m_{B S}}=\left(\frac{60 \mathrm{eV}}{m_{B S}}\right) 2 \times 10^{-5} \mathrm{~cm}
$$

In QCD one has $E_{c} \simeq 200 \mathrm{MeV}$ with the pion mass $m_{\pi} \simeq 140 \mathrm{MeV}$ while the baryons mass (protons and neutrons) $m_{b} \simeq 940 \mathrm{MeV}$, i.e. the proportionality constant is in the range $0.7<d<5$, and with bound mass much larger than the mass of the quarks $\left(m_{u} \simeq\right.$ $\left.(1-3) \mathrm{MeV}, m_{d} \simeq(3.5-6) \mathrm{MeV}\right)$. Clearly the mass of the bound states is not the sum of its elementary particles but is due to the non-perturbative effects of the strong force and is well parameterized by $E_{c}$. The dynamical formation of bound states is not completely understood since it involves non perturbative physics. However, it has been shown in RHIC [19] that at high density, above the transition scale $E_{c}$, the QCD quarks do indeed behave as free particles, while at low energies there are no free elementary quarks and all quarks form gauge neutral bound states. Since the interaction strength increases at lower energies, the formation of bound states is expected to be larger at the smallest possible particle bound state energy $E_{B S}$ (i.e. $E_{B S}=m_{B S}$ ) with momentum $p^{2}=E_{B S}^{2}-m_{B S}^{2} \simeq 0$. The energy distribution of bound states formation is still under investigation [21] and for simplicity we take here $p=0$ which gives a vanishing particle velocity for the bound states.

It is precisely the non-perturbative gauge mechanism that we have in mind for our bound states dark matter BDM. Of course, in our case the gauge group and elementary fields are not part of the SM. This "dark" gauge 
group is assumed to interact with the SM only through gravity and is widely predicted by extensions of the SM, such as brane or string theories. Furthermore, this dark gauge group may also account for dark energy as dark mesons [18]. The model used in [18] has a transition scale $E_{c}=O(10-100) \mathrm{eV}$ which is the reference energy used here. We see from eq.(25) that for $m=d E_{c}=60 \mathrm{eV}$ we have a small Compton wave length $\lambda_{c o m} \simeq 10^{-5} \mathrm{~cm}$ and we therefore do not expect it to play a relevant role in late cosmology or galactic scales.

Even tough we have motivated our BDM in terms of a well motivated particle physics model we stress the fact that the cosmological implications of BDM do not depend on its origin of. The BDM is defined by a DM that at $\rho_{b d m}>\rho_{c}$ behaves as relativistic HDM with a particle velocity $v=1$ while for $\rho_{b d m}<\rho_{c}$ it is CDM with $v \simeq 0$. Naturally one encounters high $\rho$ at early cosmological times and in the inner regions of galaxies.

\section{SUMMARY AND CONCLUSIONS}

We have presented a new type of dark matter, BDM, which can be derived from particle physics. These new particles depend on a dark gauge group, similar as the SM elementary particles, but with only gravitational interaction with the SM. The BDM particles acquire a non perturbative mass below the phase transition scale $E_{c}$. The order of magnitude for this phase transition is $E_{c}=O(10-100) e V[18]$. Above $E_{c}$ we have relativistic elementary particles while below this scale we have nonrelativistic bound states, formed as neutral bound states from the elementary fields. The cosmological effect of the phase transition manifest itself in two different regions. Firstly, in the cosmological evolution of the energy density $\rho_{b d m}$ and secondly in the inner regions of galaxies. From a cosmological evolution we have a $\rho_{b d m}$ redshifting as radiation above $\rho_{c}=E_{c}^{4}$ and as matter below this scale. Since the BDM particles are relativistic at high energies we have a nonvanishing free streaming scale $\lambda_{f s}$ with a contained mass $M_{f s}$. At the same time our BDM predicts a core galaxy profile with radius $r_{\text {core }}$ once the energy density of the galaxies $\rho_{g}$ reaches the transition scale $\rho_{c}$. The quantities $\lambda_{f s}, M_{f s}$ and $r_{\text {core }}$ are given by eqs.(8), (10) and (16) and have the same origin, namely the phase transition scale, and are determined in terms of a single parameter $E_{c}$. Therefore, we have proposed a DM model which connects the solution to two of the main shortcomings of CDM, namely the overabundance of substructure in CDM halos and the rotation curves for DM dominated galaxies.

Acknowledgment- We thank for partial support Conacyt Project 80519, IAC-Conacyt Project.
[1] SDSS Collaboration,Jennifer K. Adelman-McCarthy et al., Astrophys. J.Suppl.175:297-313,2008; The 2dFGRS Collaboration,Shaun Cole et al.,Mon.Not. Roy.Astron.Soc.362:505-534,2005

[2] ESSENCE Collaboration, W.Michael Wood-Vasey et al., Astrophys.J.666:694-715,2007; SNLS Collaboration, Pierre Astier et al., Astron. Astrophys.447:31-48,2006.; Supernova Search Team, Adam G. Riess et al., Astrophys. J.607:665-687,2004

[3] WMAP Collaboration, E. Komatsu et al, Astrophys. J.Suppl.180:330-376,2009.

[4] Navarro, J.F., Frenk, C.S., White, S.D.M. 1996, ApJ, 462, 563 Navarro, J.F., Frenk, C.S., White, S.D.M. 1997, ApJ, 490, 493

[5] Fukushige T., Makino J., 1997, ApJ, 477, L9; Moore B., Governato F., Quinn T., Stadel J., Lake G., 1998, ApJ, 499, L5; Moore B., Quinn T., Governato F., Stadel J., Lake G., 1999, MNRAS, 310, 1147; Ghigna S., Moore B., Governato F., Lake G., Quinn T., StadelJ., 1998, MNRAS, 300, 146; Klypin A.A., Gottlober S., Kravtsov A.V., Khokholov A.M.,1999, ApJ, 516, 530

[6] Kauffmann G.,White S.D.M., Guiderdoni B., 1993, MNRAS, 264, 201; Klypin A.A., Kravtsov A.V., Valenzuela O., Prada F., 1999, ApJ, 522, 82; Moore B., Ghigna S., Governato F., Lake G., Quinn T., Stadel J., Tozzi P., 1999, ApJ, 524, L19; McGaugh, S.S., Barker, M.K., de Blok, W.J.G. 2003, ApJ, 584,566; Swaters, R.A., Verheijen, M.A.W., Bershady, M.A., Andersen, D.R. 2003, ApJ, 587, 19

[7] Burkert A., 1995, ApJ, 447, L25
[8] Flores R., Primack J.R., 1994, ApJ, 427, L1; Moore B., 1994, Nature, 370, 629; Burkert A., Silk J., 1997, ApJ, 488, L55; McGaugh S.S., de Blok W.J.G., 1998, ApJ, 499, 41; Firmani C., DOnghia E., Chincarini G., Hernandez X., Avila-Reese V., 2001, MNRAS, 321, 713; Navarro, J.F., Hayashi, E., Power, C., Jenkins, A.R., Frenk, C.S., White, S.D.M., Springel, V., Stadel, J., Quinn, T.R. 2004, MNRAS, 349, 1039;

[9] de Blok, W.J.G., McGaugh, S.S. 1996, ApJ, 469, L89; Pickering, T.E., Impey, C.D., van Gorkom, J.H., Bothun, G.D.,1997, AJ, 114, 1858; Blais-Ouellette, S., Amram, P., Carignan, C. 2001, AJ, 121, 1952; Borriello, A., Salucci, P. 2001, MNRAS, 323, 285; Simon, J.D., Bolatto, A.D., Leroy, A., Blitz, L. 2003, ApJ, 596, 957

[10] F. C. van den Bosch, R.A. Swaters, 2001, MNRAS, 325,1017; R.A. Swaters, B.F. Madore, F.C. van Den Bosch, M. Balcells, Astrophys.J.583:732-751,2003.

[11] G. Gentile, P. Salucci, U. Klein, D. Vergani, P. Kalberla, 2004, MNRAS, 351, 903; Simon, J.D., Bolatto, A.D., Leroy, A., Blitz, L., Gates, E. 2005, ApJ, 621, 757; de Blok, W.J.G. 2003, IAUS, 220, 191; de Blok, W.J.G., Bosma, A. 2002, A \& A, 385, 816 (BB02); Bolatto, A.D., Simon, J.D., Leroy, A., Blitz, L. 2002, ApJ, 565, 238; Cote, S., Carignan, C., Freeman, K.C. 2000, AJ, 120, 3027 ;

[12] Navarro J.F., Eke V.R., Frenk C.S., 1996, MNRAS, 283, 72; Gelato S., Sommer-Larson J., 1999, MNRAS, 303, 321; van den Bosch F.C., Robertson B.E., Dalcanton J.J., de Blok W.J.G., 2000, AJ, 119, 1579; Bullock J.S., Kravtsov A.V., Weinberg D.H., 2000, Astro- 
phys.J.539:517, 2000; Binney J.J., Gerhard O.E., Silk J., 2001, MNRAS, 321, 471

[13] McGaugh S.S., de Blok W.J.G., 1998, ApJ, 499, 41; Strickland D.K., Stevens I.R., 2000, MNRAS, 314, 511

[14] Kamionkowski M., Liddle A.R., 2000, Phys. Rev. Let. 84, 4525; White M., Croft R.A., 2000, ApJ, 539, 497

[15] Avila-Reese V., Colin P., Valenzuela O., D'Onghia E., Firmani C., Astrophys. J.559:516-530,2001. SommerLarsen J., Dolgov A., Astrophys.J.551:608-623,2001 Hogan C.J., Dalcanton J.J., Phys.Rev.D62:063511, 2000.

[16] Peebles P.J.E., Vilenkin A., 1999, Phys. Rev. D., 60, 103506; Hu W., Peebles P.J.E., Astrophys. J.528:L61L64,2000; Matos T., Siddharta G.F., Urena-Lopez L.A., 2000, Clas. Quantum Grav. 17, 1707

[17] Carlson E.D., Machacek M.E., Hall L.J., 1992, ApJ, 398, 43; Spergel D.N., Steinhardt P.J., 2000, Phys.
Rev. Let. 84, 17; Mohapatra R.N., Teplitz V.L., Phys.Rev.D62:063506, 2000; Firmani C., DOnghia E., Avila-Reese V., Chincarini G., Hernandez X., 2000, MNRAS, 315, L29; Kaplinghat M., Knox L., Turner M.S., 2000, Phys. Rev. Let. 85; Bento M.C., Bertolami O., Rosenfeld R., Teodoro L., Phys.Rev.D62:041302,2000

[18] A. de la Macorra, JHEP 0301:033, 2003; A. de la Macorra, Phys.Rev.D72:043508, 2005; A. de la Macorra, Phys.Lett.B585, 2004

[19] STAR Collaboration,John Adams et al., Nucl.Phys.A757:102-183,2005; BRAHMS Collaboration, I. Arsene et al., Nucl.Phys.A757:1-27,2005

[20] Particle Data Group,C. Amsler et al., Phys.Lett.B667:1,2008

[21] A. Bazavov et al., arXiv:0903.4379 